\title{
DETERMINATION OF SOME PHYSICAL PARAMETERS \\ OF THE MOON WITH LUNAR LASER RANGING DATA
}

\author{
JIN WENJING AND LI JINLING \\ Shanghai Observatory \\ Shanghai, P.R. China
}

\begin{abstract}
Information about the structure of lunar interior and evolution could be obtained from measurements of lunar free librations, gravitational field, dissipation etc. In this paper the precision of determining free librations from Lunar Laser Ranging (LLR) data are estimated. Using the observing data from four telescopes for eighteen years, the amplitudes and phases of free librations, and ratios of the moments of inertia of the Moon were determined.
\end{abstract}

\section{Introduction}

The information on lunar physics is mainly obtained from the lunar seismometer network, which was formed by Apollo 12, 14, 15, 16 missions and from analysing the soil and rock brought by astronauts. The gravitational field and moments of inertia of the Moon are usually determined by lunar orbiters.

Since 1976 lunar probes have not been launched owing to financial problems. The network of lunar laser ranging has been developed from one station to three. Meanwhile, to range four retroreflectors becomes a regular observing procedure. With the development of advanced techniques the pulse width becomes narrower, from $2.5 \mathrm{~ns}$ to $100 \mathrm{ps}$, so the precision of ranging the Moon is enhanced from $18 \mathrm{~cm}$ to $5 \mathrm{~cm}$. The precision of determining lunar free librations and the third-degree gravitational harmonics has been improved.

\section{Data Used}

The returned photons from retroreflectors were received at $\mathrm{McDonald} \mathrm{Ob}$ servatory with the 2.7 meter telescope in August of 1969. Starting from

I. M. Wytrzyszczak, J. H. Lieske and R. A. Feldman (eds.),

Dynamics and Astrometry of Natural and Artificial Celestial Bodies, 221, 1997.

(C) 1997 Kluwer Academic Publishers. Printed in the Netherlands. 


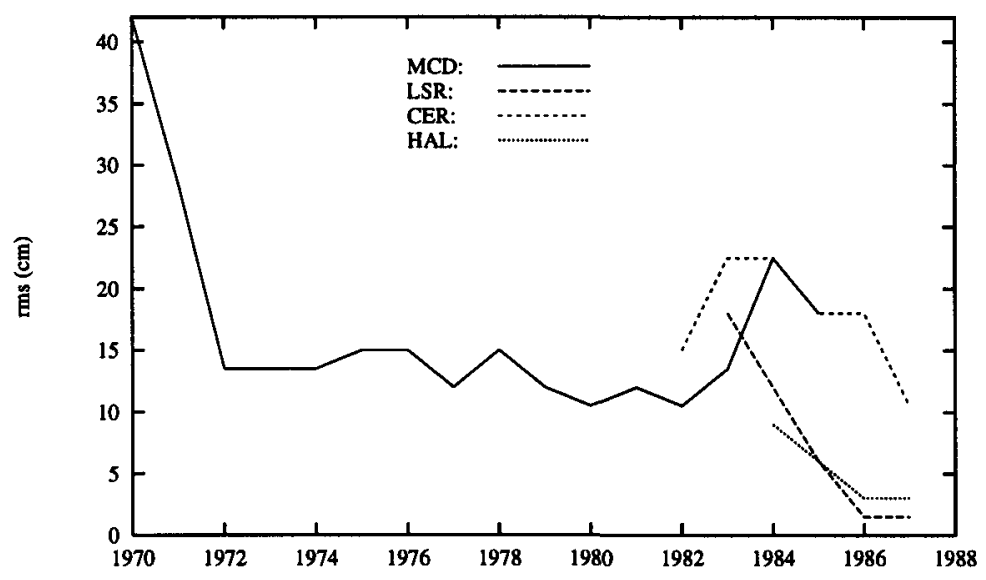

Figure 1. Precision of normal points for each station.

1970 to 1981 , only one station in the world had carried out lunar laser ranging regularly. During the period August - October 1980 and September 1983 - October 1984, the program of observation and analysis called Monitoring Earth Rotation and Intercomparing the Techniques (MERIT) was carried out. MERIT pushed the development and routine observations of Very Long Baseline Interforemetry (VLBI), Satellite Laser Ranging (SLR) and LLR in the world (Muller, 1985). Since 1983 there are three stations doing lunar laser ranging. The precision of normal points for each station is shown in Figure 1. The codes of stations and reflectors used in this paper are listed in Table 1.

TABLE 1. The codes of station and reflector.

\begin{tabular}{ll}
\hline \multicolumn{1}{c}{ Station } & \multicolumn{1}{c}{ Reflector } \\
\hline MCD=McDonald $2.7 \mathrm{~m}$ & TRA=Tranquility \\
LRS=MLRS & FRM=Fra Mauro \\
HAL=Haleakala & HAD=Hadley \\
CER=CERGA & LK2=Lunakhod 2 \\
\hline
\end{tabular}

It is well known that the libration parameters could not be obtained from observations of a single reflector (Mulholland, 1978). Statistics of normal points from global observing network during 1970 to 1987 shows that the number of normal points for each reflector is not well distributed. The ratios for different reflectors are TRA 9.5\%, FRM $10.8 \%$, HAD $75.4 \%$ and $\mathrm{LK}_{2}$ $4.3 \%$ respectively. Therefore it is disadvantageous for measuring the free librations. 


\section{Precision of Determining Free Librations}

On the surface of the Moon there are five retroreflectors among which three reflectors were installed by American astronauts during Apollo missions and others were made in France and put on by Russian cosmonauts.

TABLE 2. Selenographic coordinates of reflectors.

\begin{tabular}{lcrr}
\hline & R $[\mathrm{km}]$ & longitude [deg] & latitude [deg] \\
\hline TRA & $\mathbf{1 7 3 5 . 4 7 7 0 7 3}$ & 23.45093088 & 0.69352820 \\
FRM & $\mathbf{1 7 3 6 . 3 3 9 0 5 0}$ & -17.50041767 & -3.62321101 \\
HAD & 1735.481089 & 3.60702873 & 26.15530489 \\
LK $_{2}$ & 1734.642539 & 30.90537743 & 25.85105146 \\
\hline
\end{tabular}

Since there is a departure of the spin axis from the major axis of inertia, forced and free oscillations exist. Forced oscillations of physical librations are mainly driven by the torque exerted on the nonspherical mass distribution of the Moon by the mass of the Earth and the tidal deformation of elastic Earth (Mulholland, 1978). Now the forced physical librations are usually handled by mathematical modelling and numerical integration such as Eckhardt-500, LLB-13 and so on. The precision of these ephemerides to express the forced physical librations has attained better than $0{ }^{\prime \prime} 001$ (Moons, 1982; Newhall, 1983).

In order to estimate the measuring precision of free librations it is clear that the relation between the variations of selenographic coordinates and the variation of the distance from station to reflector can be expressed by the following formula ( $\mathrm{Li}$ et al., 1989),

$$
\begin{aligned}
\Delta \rho & =(\cos \phi \sin \lambda \Delta \lambda+\cos \lambda \sin \phi \Delta \phi) R S / \rho \\
\Delta \lambda & =\Delta L-\tan \phi \sin \lambda \Delta F \\
\Delta \phi & =-\cos \lambda \Delta F
\end{aligned}
$$

where

$\Delta \rho$ is the variation of distance between the observing station and measuring reflector,

$S$ is the distance between the geocenter and the center of mass of the Moon,

$R, \lambda, \phi$ are selenographic coordinates of reflector,

$\Delta L, \Delta F$ are rotations about the $X_{3}$ and $X_{2}$ axes to express the physical librations in longitude and latitude.

Here axis 1 is in the mean direction of the Earth. Axis 3 is in the direction of the lunar north pole and axis 2 is orthogonal to axes 1 and 3 to complete a righthanded system. 
According to the observational precision of lunar laser ranging and the ratio of the numbers of normal points for each reflector, the precision of $\Delta L$ and $\Delta F$ estimated with Equation 1 are listed in Table 3.

TABLE 3. Estimated precision of free librations.

\begin{tabular}{ccccc}
\hline & \multicolumn{2}{c}{$18 \mathrm{~cm}$} & \multicolumn{2}{c}{$5 \mathrm{~cm}$} \\
\hline Relative weight & $\begin{array}{c}\text { longitude } \\
{\left[{ }^{\prime \prime}\right]}\end{array}$ & $\begin{array}{c}\text { latitude } \\
{\left[{ }^{\prime \prime}\right]}\end{array}$ & $\begin{array}{c}\text { longitude } \\
{\left[{ }^{\prime \prime}\right]}\end{array}$ & $\begin{array}{c}\text { latitude } \\
{\left[{ }^{\prime \prime}\right]}\end{array}$ \\
\hline $1: 1: 1: 1$ & 0.016 & 0.018 & 0.004 & 0.005 \\
$1: 1: 1.4: 1$ & 0.016 & 0.016 & 0.004 & 0.004 \\
$9.5: 10.8: 75.4: 4.3$ & 0.025 & 0.012 & 0.007 & 0.003 \\
\hline
\end{tabular}

From Table 3 it is clear that the physical libration in longitude could be determined by ranging to the reflectors TRA and FRM and that in latitude by ranging to reflectors HAD and TRA (or HAD and FRM). According to the present distribution of reflectors the optimum observing schedule is that the number of normal points for ranging to reflector HAD is about 1.5 times of that for ranging to others. Based on the present precision of lunar laser ranging the precision of free librations about 0.005 can be obtained.

\section{Determination of Lunar Free Librations and Dissipation}

Free oscillations of the Moon, so called free librations, which are not forced, are caused by the spin axis not coinciding with major inertia axis. The amplitudes and phases of free librations can be completely determined by the observations of lunar laser ranging. The theoretical modes of free librations can be expressed as follows (Calame, 1976):

$$
\begin{aligned}
& \tau=A \sin (\omega t+g) \\
& p_{1}=A_{1} \sin \left(\omega_{1} t+g_{1}\right)+A_{2} \sin \left(\omega_{2} t+g_{2}\right) \\
& p_{2}=K_{1} A_{1} \cos \left(\omega_{1} t+g_{1}\right)+K_{2} A_{2} \cos \left(\omega_{2} t+g_{2}\right)
\end{aligned}
$$

in which

$\tau$ is the free libration in longitude,

$p_{1}, p_{2}$ are direction consines of the ecliptic pole,

$A_{1}, A_{2}$ are amplitudes of free librations, and

$\omega=5.992811419 \times 10^{-3} \mathrm{rad} / \mathrm{day}$,

$\omega_{1}=0.230184429 \mathrm{rad} /$ day,

$\omega_{2}=2.303155338 \times 10^{-4} \mathrm{rad} / \mathrm{day}$,

$K_{1}=0.9990713$,

$K_{2}=-2.4872105$. 
From the lunar laser ranging data, which spanned the interval from April 1970 to December 1987, we estimated simultaneously by a weighted least squares adjustment up to 55 parameters, including twelve station coordinates, twelve selenographic coordinates of reflectors, two lunar moments of inertia ratios, seven third- degree harmonics of lunar gravitational field, ten orbital elements of the Moon and the Earth, lunar tidal acceleration, the Nordvedt term, six free librations and four bias parameters to be accounted in the ranging observations. Free librations obtained from some researchers with different methods are listed in Table 4 . As shown in Table 4 the results

TABLE 4. Amplitudes and phases of free librations.

\begin{tabular}{cccccc}
\hline Author & Calame & Calame & $\begin{array}{c}\text { Calame\& } \\
\text { Mulholland }\end{array}$ & Yoder & This paper \\
\hline Data & $1969.8-$ & $1969.8-$ & & & $\begin{array}{r}1970.4- \\
\text { span }\end{array}$ \\
1974.11 & 1975.12 & & & 1987.12 \\
\hline Data & LLR & LLR & Meteorite & Core- & LLR \\
& & & impacts & mantle & \\
\hline A['] & $1.7 \pm 0.1$ & $1.8 \pm 0.1$ & $0.2-4.6$ & 1. & $1.865 \pm 0.007$ \\
g[deg] & $232.8 \pm 5$ & $239 . \pm 5$. & $/$ & $/$ & $238.2 \pm 0.2$ \\
$A_{1}\left[{ }^{\prime \prime}\right]$ & $0.5 \pm 0.2$ & $0.4 \pm 0.2$ & 0.14 & 0.4 & $0.374 \pm 0.004$ \\
$g_{1}[\mathrm{deg}]$ & $25 . \pm 20$. & $354 . \pm 20$. & $/$ & $/$ & $315.9 \pm 0.7$ \\
$A_{2}\left[{ }^{\prime \prime}\right]$ & $3.5 \pm 1.0$ & $3.1 \pm 1.0$ & 0.2 & 2.7 & $3.249 \pm 0.011$ \\
$g_{2}[\mathrm{deg}]$ & $343.8 \pm 1.0$ & $313 . \pm 10$. & 15.7 & $/$ & $319.1 \pm 0.3$ \\
\hline
\end{tabular}

are consistent with each other. The precision of our results is higher than that obtained by Calame because the span of data is three times longer than that used by Calame $(1976,1977)$. Calame and Mulholland (1978) and Yoder (1981) had explained that the plausible mechanisms of free librations were the meteorite impact at Gordano Brano $\left(103^{\circ}\right.$ East, $36^{\circ}$ North $)$ on the surface of the Moon in the year of 1178 and the turbulent core-mantle friction respectively. Although the results calculated with different hypotheses are consistent with the determinations of the free librations from lunar laser ranging, it is hard to say that the meteorite impact is the continuous excitation source. Most scientists are in favor of the latter hypothesis since it seems more reasonable as a continuous cause of excitation.

Suppose the free librations are the pure resistance oscillations. Their models could be expressed as follows:

$$
\begin{aligned}
& \tau=A e^{-\omega t / 2 Q} \sin (\omega t+g) \\
& p_{1}=A_{1} e^{-\omega_{1} t / 2 Q_{1}} \sin \left(\omega_{1} t+g_{1}\right)+A_{2} \sin \left(\omega_{2} t+g_{2}\right)
\end{aligned}
$$


where, $A, \omega, g$ and $Q$ denote the amplitude, angular frequency, phase and dissipation factor. The results from data reduction are

$$
\begin{array}{llll}
A=1.855 & \omega=5.991411419 \times 10^{-3} \mathrm{rad} / \text { day } & Q=9720 & g=240.3 \\
A_{1}=0.377 & \omega_{1}=0.230184429 \mathrm{rad} / \text { day } & Q_{1}=3650 & g_{1}=346.4
\end{array}
$$

There is a considerable inherent difficulty for determining the other mode of free libration in latitude because the observations were only 25 percent of the period. The results of dissipation factor, being very large, are consistent with those obtained from analysing the data of moonquake (Toksöz et al., 1974). It can be seen that the free oscillation of the Moon is very similar to the Chandler Wobble of the Earth. The procedure of excitation and dissipation will be discussed in further research.

In conclusion as analysed above the free librations of the Moon, like the Chandler Wobble of the Earth, exist. The amplitudes and phases of free librations determined by the authors of this paper are consistent with those obtained by others.

\section{References}

Calame, O.: 1976, "Free librations of the Moon determined by an analysis of laser ranging measurements", The Moon 15, 343.

Calame, O.: 1977, "Free librations of the Moon from lunar laser ranging", in: Scientific Application of LLR (J.D. Mulholland, ed.), Reidel, Dordrecht, p.53.

$\mathrm{Li}$, Jinling, Nie, Zhaoming, and Jin Wenjing: 1989, "The optimum distribution of the retroreflectors for the determination of the physical libration of the Moon", Publications of Yunnan Observatory, No 3, 1.

Standish, E.M. et al.: 1995, JPL Planetary and Lunar Ephemerides DE403/LE403, JPL IOM 314.10-127.

Moons, M.: 1982, "Physical libration of the Moon", Celest. Mech. 126, 131.

Mulholland, J.D.: 1978, "Lunar carter Giordano: A.D.1178 impact observations consistent with laser ranging results", Science 199, 875.

Muller, I.I.: 1985, Proceedings of the International Conference on Earth Rotation and the Terrestrial Reference Frame.

Newhall, X X: 1983, "DE102: A numerically integrated ephemeris of the Moon and planets spanning forty-four centuries", Astron. Astrophys. 125, 150.

Solomon, S.C.: 1974, "Density within the Moon and implications for lunar composition", The Moon 9, No $1 / 2,147-166$.

Toksöz, M.N., Dainty, A.M., and Solomon, S.C.: 1974, "A summary of lunar structural constraints", The Moon 10, No 3/4, 370-371.

Williams, J.G., Newhall, X X, and Dickey, J.O.: 1986, "Lunar gravitational harmonics and reflector coordinates", JPL Geodesy and Geophysics Preprint, No 150.

Yoder, C.F.: 1981, "The free librations of a dissipative Moon", Phil. Trans. R. Soc. Lond. A 303, 327-338. 